\title{
Модельная среда с тепловыми и транспортными свойствами жидкой воды
}

\author{
(C) А.А. Васин, А.А. Волков \\ Институт общей физики им. А.М. Прохорова РАН, \\ 119991 Москва, Россия \\ e-mail: alexcampeones@yandex.ru
}

Поступило в Редакцию 15 декабря 2019 г.

В окончательной редакции 15 декабря 2019 г.

Принято к публикации 17 февраля 2020 г.

Рассмотрена модель френкелевской газо-твердотельной среды, состоящей из молекул $\mathrm{H}_{2} \mathrm{O}$ и ионов $\mathrm{H}_{3} \mathrm{O}^{+}$и $\mathrm{OH}^{-}$, имеющей одинаковую с жидкой водой плотность и концентрацию частиц. Частицы совершают тепловое колебательно-диффузионное движение, в процессе которого сталкиваются, обмениваются протонами и взаимопревращаются. В предположении, что среда удерживается силами диполь-ионного взаимодействия, показано, что энтальпия испарения $H$, теплоемкость $C$, а также транспортные параметры среды коэффициент самодиффузии $D$, вязкость $\eta$ и теплопроводность $\theta-$ близки к справочным для жидкой воды во всем интервале ее существования $(250-600 \mathrm{~K})$.

Ключевые слова: вода, модель, энтальпия испарения, самодиффузия, вязкость, теплопроводность.

DOI: $10.21883 / J T F .2020 .09 .49677 .404-19$

\section{Введение}

Свойства жидкой воды, несмотря на их долгое и интенсивное изучение, в полной мере не поняты и считаются аномальными. Проблеме посвящен тематический сборник 2016 года „Water — The Most Anomalous Liquid“ [1]. Для достижения понимания актуален поиск простых и физически ясных моделей: „Finding simple water models reproducing as many properties as possible constitutes an important subject of research" [2]. В нашем поиске [3,4] мы обратились к идее Френкеля о газотвердотельном диффузионно-колебательном характере движения молекул в жидкостях [5]. С опорой на литературные данные [6] мы пришли к выводу, что свойства воды формируют на равных правах частицы трех типов - молекулы $\mathrm{H}_{2} \mathrm{O}$ и ионы $\mathrm{H}_{3} \mathrm{O}^{+}$и $\mathrm{OH}^{-}$, которые совершают свободное тепловое движение, сталкиваются, обмениваются протонами и взаимопревращаются. Столкновительная передача протонов ведет к тому, что в среде надмолекулярно броуновски блуждают положительные и отрицательные заряды. Система зарядов имеет собственную степень свободы движения.

Модель противоречит принятому представлению о воде, как об ансамбле неделимых молекул $\mathrm{H}_{2} \mathrm{O}$, связанных плотной сеткой водородных связей [7], однако модель последовательна, непротиворечива и заслуживает проверки. Ниже мы анализируем тепловые и транспортные свойства описанной среды и находим их близкими к тем, которые присущи реальной жидкой воде.

\section{1. Ион-молекулярная модель среды}

Рассматривается среда, изображенная на рис. 1, с геометрическими параметрами жидкой воды: молекула- ми-кружками диаметром $d=2.8 \square$, разделенными расстоянием в четверть диаметра $\delta=0.7 \square$ [6]. Среда представляет собой плотный газ частиц, состоящих из атомов кислорода с распределенными по ним случайным образом протонами: частица с двумя протонами нейтральная молекула $\mathrm{H}_{2} \mathrm{O}$, а с 1 и 3 протонами ионы $\mathrm{H}_{3} \mathrm{O}^{+}$и $\mathrm{OH}^{-}$. В результате тепловых соударений количество протонов в составе частицы изменяется, положительные и отрицательные заряды хаотически переходят от частицы к частице.

В основу динамики среды закладываем следующие постулаты.

1. В среде поддерживается зарядовый баланс:

$$
\left(N_{0}-N_{i}\right) / \tau_{D}=N_{i} / \tau_{i}
$$

где $N_{0}=3.3 \cdot 10^{28} \mathrm{~m}^{-3}-$ полная концентрация частиц $\left(N_{0}=\rho / m\right.$, где $\rho=1 \mathrm{~kg} / \mathrm{dm}^{3}$ - плотность воды и $m=3 \cdot 10^{-26} \mathrm{~kg}$ - масса молекулы $\left.\mathrm{H}_{2} \mathrm{O}\right), N_{i}-$ суммарная концентрация ионов $\mathrm{H}_{3} \mathrm{O}^{+}$и $\mathrm{OH}^{-}, \tau_{D}$ и $\tau_{i}-$ времена жизни нейтральных и заряженных частиц.

2. Среднее расстояние между ионами $L$ связано с концентрацией $N_{i}$ условием плотной упаковки сфер:

$$
L=\left(2^{1 / 2} / N_{i}\right)^{1 / 3} \text {. }
$$

3. Ионы движутся по закону броуновской диффузии с пространственным параметром $L$, поддерживающим восстановление структуры ионов. C $L$ сопрягается временной параметр $\tau$, время восстановления ионной структуры. Это время принимается равным времени дебаевской релаксации $\tau_{D}$. Коэффициент самодиффузии выглядит как $D=L^{2} /\left(12 \pi \tau_{D}\right)$ (соотношение Смолуховского-Эйнштейна $[8])$ или с учетом (2), как

$$
D=2^{1 / 3} /\left(12 \pi N_{i}^{2 / 3} \tau_{D}\right) .
$$


4. Дебаевская релаксация в воде, которая традиционно интерпретируется на языке диэлектрической проницаемости [9] в форме

$$
\varepsilon(\omega)=\varepsilon_{\infty}+\Delta \varepsilon_{D}\left(1+i \omega \tau_{D}\right),
$$

где $\Delta \varepsilon_{D}$ - диэлектрический вклад и $\varepsilon_{\infty}-$ суммарный вклад высокочастотных механизмов поляризации рассматривается в терминах динамической проводимости $\sigma^{*}(\omega)=i \omega \varepsilon_{0} \varepsilon^{*}(\omega)$, где $\varepsilon_{0}=8.85 \cdot 10^{-12} \mathrm{~F} / \mathrm{m}$ - диэлектрическая проницаемость вакуума.

5. На частотах, выше дебаевской частоты $1 / \tau_{D}$, проводимость выходит на плато:

$$
\sigma_{D}(\infty)=\varepsilon_{0} \Delta \varepsilon_{D} / \tau_{D}
$$

6. Предельная проводимость (5) считается электродинамическим отражением диффузи (3), и они связываются друг с другом соотношением Нернста-Эйнштейна [8]:

$$
\sigma_{D}(\infty)=q^{2} N_{i} D /\left(k_{B} T\right),
$$

где $q=1.6 \cdot 10^{-19} \mathrm{C}-$ элементарный заряд и $k_{B}=1.38 \cdot 10^{-23} \mathrm{~J} / \mathrm{K}$ - константа Больцмана.

В работах $[3,4]$ нами показано, что система уравнений (1)-(6) исчерпывающе описывает диэлектрические спектры воды $\varepsilon^{\prime}(\omega)$ и $\sigma(\omega)$ при комнатной температуре до частот $10^{13} \mathrm{~Hz}$. Ниже мы распространяем этот подход на тепловые и транспортные свойства среды.

\section{2. Тепловые свойства среды}

На рис. 1 обращаем внимание на молекулярный мотив в виде иона, окруженного нейтральными молекулами $\mathrm{H}_{2} \mathrm{O}$. Четыре таких иона в клетке из соседей представлены в углах рисунка. При столкновении иона с нейтральной молекулой клетки лишний протон (или дырка от протона) может сменить хозяина (атом кислорода). Протон (дырка) перемещается тогда на границу клетки. Вместе с ним перемещается заряд, а за зарядом подтягивается поляризационная конфигурация диполей. В конечном счете, конструкция „ион в клетке из соседей“ воспроизводится на новом месте. С шагом в размер межкислородного расстояния $l$ конструкция совершает броуновскую диффузию.

Скорость преобразования структуры связана с временем пролета молекулой межмолекулярного расстояния $\delta=0.7 \square$, так, что при тепловой скорости молекулы воды $\sim 640 \mathrm{~m} / \mathrm{s}$ (при комнатной температуре) молекулярная динамика разворачивается в масштабе пикосекундных времен. При больших временах усреднения дипольные молекулы $\mathrm{H}_{2} \mathrm{O}$ оказываются постоянно находящимися в полях притяжения однозарядных ионов $\mathrm{H}_{3} \mathrm{O}^{+}$ и $\mathrm{OH}^{-}$. Средняя энергия притяжения $E_{q p}$ выражается формулой электростатического ион-дипольного взаимодействия [10]. Умноженная на концентрацию частиц эта энергия $H=E_{q p} N_{0}$ является энергией связи всего ансамбля:

$$
H=N_{0} E_{q p}=N_{0} q p /\left(4 \pi \varepsilon_{0} l^{2}\right)
$$

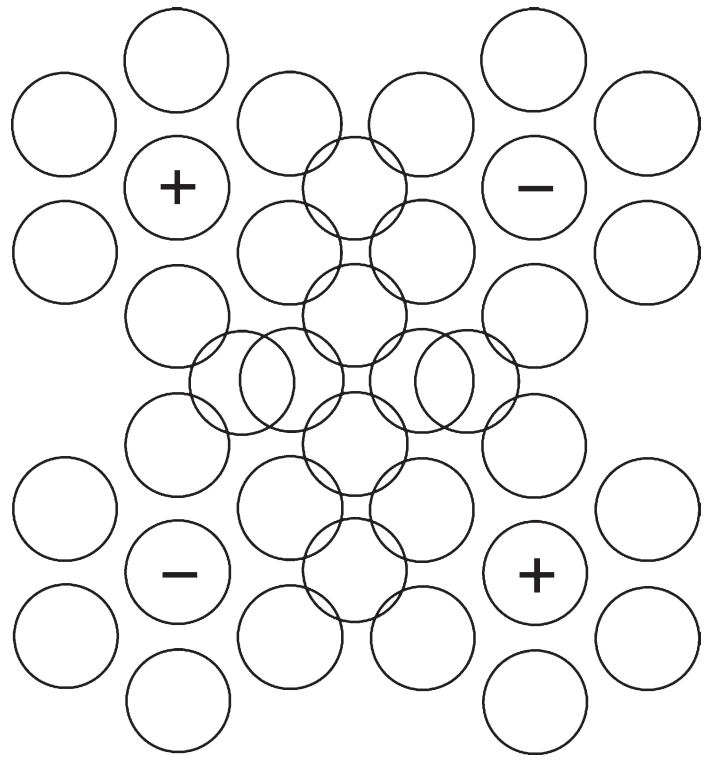

Рис. 1. Ион-молекулярная модель жидкой воды. Пустые кружки - нейтральные молекулы $\mathrm{H}_{2} \mathrm{O}$, кружки с плюсами - ионы $\mathrm{H}_{3} \mathrm{O}^{+}\left(\mathrm{H}_{2} \mathrm{O}\right.$ с третьим лишним протоном), с минусами ионы $\mathrm{OH}^{-}$(с одним недостающим протоном). Наслоение кружков в центре рисунка символизирует трехмерность среды.

где $q=1.6 \cdot 10^{-19} \mathrm{C}-$ элементарный заряд, $p=6.14 \cdot 10^{-30} \mathrm{Cm}$ - дипольный момент молекулы $\mathrm{H}_{2} \mathrm{O}, l-$ межкислородное расстояние $\left(3.5-10^{-10} \mathrm{~m}\right.$ при комнатной температуре [6]). Величины $l$ и $N_{0}$ предполагаем связанными условием плотной упаковки cфер $l=\left(2^{1 / 2} / N_{0}\right)^{1 / 3}$.

Энергию $H$ считаем способной в динамике конвертироваться в кинетическую энергию колебаний иона в клетке из окружающих его центрально-поляризованных молекул $\mathrm{H}_{2} \mathrm{O}$. Проявлением этого механизма считаем инфракрасный пик поглощения на частоте $5 \mathrm{THz}$ [4]. Энергию колебаний выражаем классической формулой

$$
Q=(3 / 2) N_{0} \kappa \delta^{2},
$$

где $\kappa=m \omega_{0}^{2}-$ константа упругости осциллятора, $m=3 \cdot 10^{-26} \mathrm{~kg}$ - масса атома кислорода, $\omega_{0}=2 \pi v_{0}$ $\left(v_{0}=5 \cdot 10^{12} \mathrm{~Hz}\right.$ - частота осциллятора $)$, и $\delta=l-d-$ амплитуда осцилляций.

Учтем составной характер осциллятора и взаимопревращение молекул и ионов, для чего вводим два постулата: 1) увеличиваем в формуле (8) число степеней свободы осциллятора от стандартных 3 до $n$ (под $n$ понимаем число частиц, задействованных в работе осциллятора) и 2) раскладываем энергию $H$, в соответствии с принципом равнораспределения энергии по степеням свободы, поровну между осциллирующими ионами и находящимися в поле их притяжения дипольными молекулами $\mathrm{H}_{2} \mathrm{O}$. На основании сказанного рассматриваем энергию $H$ как сумму энергий 5-THz осцилляторов и записываем ее в форме двух равных по величине 


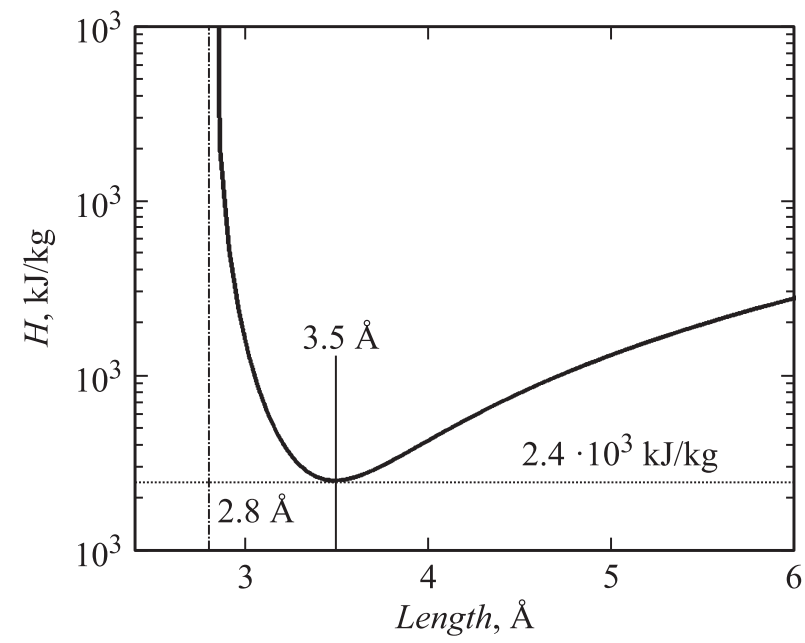

Рис. 2. Энергия связи ансамбля взаимопревращающихся заряженных и дипольных частиц в зависимости от расстояния между центрами частиц, рассчитанная по формуле (9).

слагаемых:

$$
H=N_{i}\left[q p /\left(8 \pi \varepsilon_{0} \delta^{2}\right)+n \kappa \delta^{2} / 4\right],
$$

где $N_{i}$ - концентрация ионов такая, что $n N_{i}=N_{0}$.

График зависимости $H(l)$ по формуле (9) представлен на рис. 2. Он демонстрирует хорошо выраженный минимум. Параметры минимума - положение по $l$ и минимальное значение $H-$ близки по величинам соответствующим параметрам жидкой воды - расстоянию между атомами кислорода $3.5 \square$ и энтальпии испарения $2400 \mathrm{~kJ} / \mathrm{kg}$ (при комнатной температуре) [6].

Второе слагаемое в формуле (9), растущее с ростом амплитуды осцилляций $\delta$, считаем ответственным за накопление тепла $Q$. В таком предположении производная $Q$ по температуре представляет собой теплоемкость, которая после преобразований приводится к виду [11]:

$$
C=\pi^{2} N_{0} k_{B}
$$

\section{3. Транспортные свойства среды}

Диффузию в модели совершают частицы двух разных знаков. Движение считаем подобным исследованному в работе [12]. На этом основании диэлектрический отклик в форме (4) рассматриваем как результат решения уравнения движения (сильно заторможенного) частицы массой $m$ с зарядом $q$ под действием поля $E$ в параболическом потенциале крутизной $\kappa[3]$ :

$$
m \gamma \dot{x}+k x=q E,
$$

где частота соударений, $\gamma$ - упругая константа потенциала. В измененной форме уравнение можно записать как $\dot{x}+x / \tau_{D}=\mu E$, где $1 / \tau_{D}=\kappa / m \gamma-$ характерная частота релаксации и $\mu=q / m \gamma-$ подвижность частицы.
В такой записи фактор торможения $m \gamma$ напрямую связан с коэффициентом самодиффузии $D: m \gamma=k_{B} T / D$.

Сопрягаем с учетом размерности тормозящий фактор с вязкостью $\eta: m \gamma \sim \eta L$ и после преобразований по формулам (1)-(6) получаем

$$
\eta_{m}=12 \pi k_{B} T N_{i}^{4 / 3} \tau_{D}\left(2^{1 / 2} N_{0}^{1 / 3}\right) .
$$

Подобным образом из того же набора параметров складываем выражение для теплопроводности:

$$
\theta=N_{i}^{7 / 6}\left[T\left(12 \pi k_{B}\right)^{3} /\left(2^{2 / 3} N_{0} m\right)^{1 / 2}\right]^{1 / 2} .
$$

Другие возможные варианты выражений для $\eta$ и $\theta$, подходящие по размерности, не рассматриваем, так как они на порядки величин проигрывают выражениям (12) и (13) при сравнении расчета с экспериментом.

Для полноты картины выражаем через концентрацию ионов $N_{i}$ электродинамический параметр $\Delta \varepsilon-$ диэлектрический вклад в статическую диэлектрическую проницаемость дебаевской релаксации, полученный нами ранее в работе [13]:

$$
\Delta \varepsilon_{D}=\left(2 N_{i}\right)^{1 / 3} q^{2} \beta /\left(12 \pi k_{B} T\right),
$$

где $\beta \sim 2-$ коэффициент, учитывающий эстафетный характер переноса протонов.

\section{4. Обсуждение}

На рис. 3 и 4 все расчетные кривые (3), (7), (10), (12), (13) и (14) представлены в сравнении с экспериментальными данными для жидкой воды. Данные по $H$ и $C$ взяты

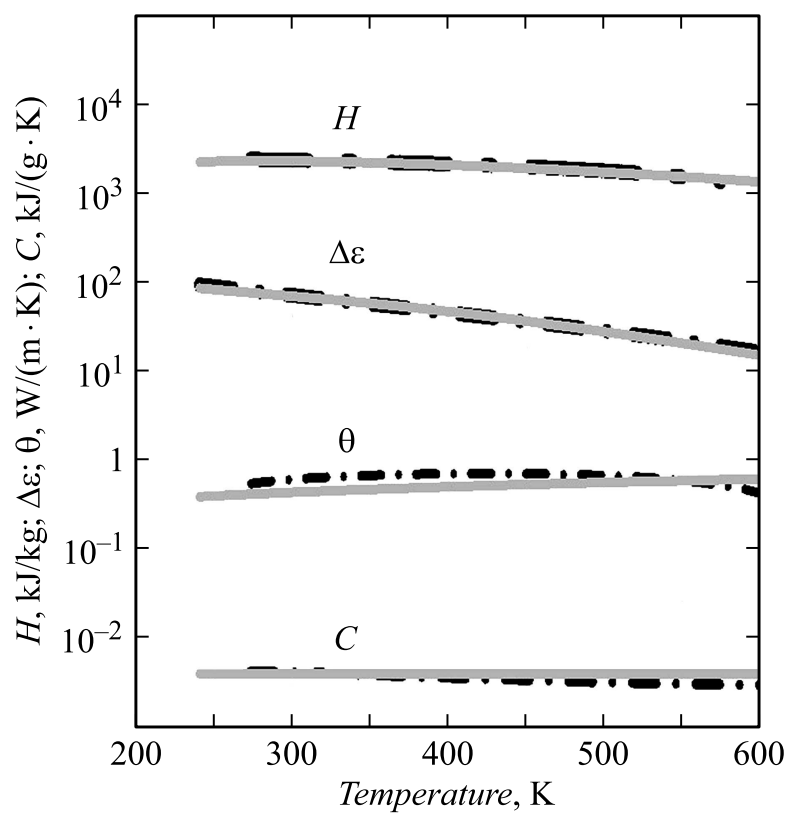

Рис. 3. Температурные зависимости энтальпии испарения $H$, диэлектрического вклада $\Delta \varepsilon$, теплопроводности $\theta$ и теплоемкости $C$. Черные линии - эксперимент для жидкой воды [14], серые линии - расчет сверху вниз по формулам соответственно (7), (14), (13) и (10) с общим для всех кривых $N_{i}=2.3 \cdot 10^{27} \mathrm{~m}^{-3}$. 


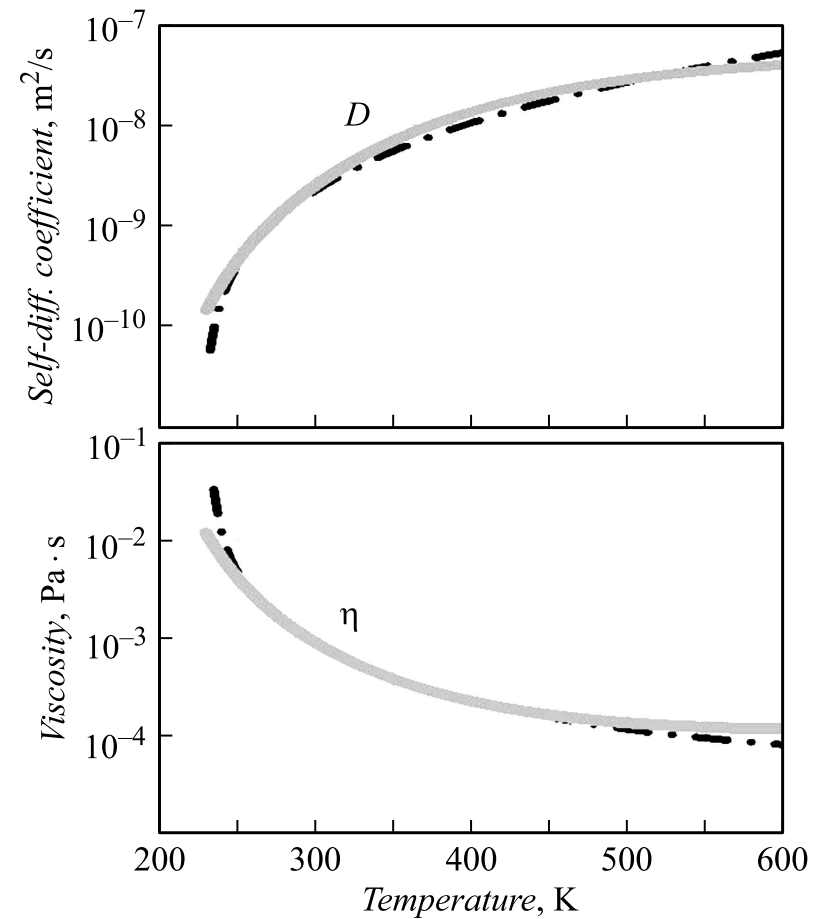

Рис. 4. Температурные зависимости коэффициентов диффузии $D$ и вязкости $\eta$. Черные линии - эксперимент для жидкой воды [14], серые линии - расчет сверху вниз по формулам, соответственно, (3) и (12) с общим для обеих кривых $N_{i}=2.3 \cdot 10^{27} \mathrm{~m}^{-3}$.

из базы [11]; по $D, \eta, \theta$ и $\Delta \varepsilon-$ из релизов [14], и по $\tau_{D}-$ из работы [15]. Общей величиной для выражений (3), (12), (13) и (14) найдена независящая от температуры концентрация ионов $N_{i}=(2 \pm 1) \cdot 10^{27} \mathrm{~m}^{-3}$. Все расчетные кривые, как видно, хорошо ложатся на экспериментальные кривые в широком диапазоне температур.

Из совпадения расчетной $H$ с энтальпией испарения воды заключаем, что ответственным за удержание молекул $\mathrm{H}_{2} \mathrm{O}$ является ион-дипольное взаимодействие взаимопревращающихся ионов и молекул. В этом случае принятая для воды динамика сетки водородных связей заменяется столкновительной динамикой молекул и ионов. Связующая роль водородных связей переходит к кулоновским полям блуждающих зарядов, порожденных лишними или недостающими протонами в составе молекул $\mathrm{H}_{2} \mathrm{O}$. На временах, больших $\tau_{D}$, быстро переходящие от молекулы к молекуле протоны обобществлены. Связь составляющих среду частиц родственна металлической (с обобществленными электронами).

Модель предполагает очень высокую концентрацию ионов, $N_{i} \sim 10^{27} \mathrm{~m}^{-3}$, и одновременно очень короткие времена жизни ионов $\left(\tau_{i} \sim 10^{-12} \mathrm{~s}\right)$ и нейтральных молекул $\mathrm{H}_{2} \mathrm{O}\left(\tau_{D} \sim 10^{-10} \mathrm{~s}\right)$. Этим модель противоречит принятому представлению, согласно которому концентрация ионов на 7 порядков меньше, а время жизни молекулы воды намного больше ( $10 \mathrm{~h} \mathrm{[16]).}$
Гигантская разница принятой для воды и полученной нами величин $N_{i}$ объясняется тем, что в предлагаемой модели по-другому, чем принято, интерпретируется спектр поглощения жидкой воды. В терминах проводимости $\sigma$ спектр поглощения воды (4) выглядит как ступенька с двумя горизонтальными полками - нижней $\sigma_{d c}\left(10^{1}-10^{7} \mathrm{~Hz}\right)$ и верхней $\sigma_{a c}\left(10^{10}-10^{13} \mathrm{~Hz}\right) \quad$ [3]. Со времен Дебая концентрация ионов $N_{i}$ неизменно исчисляется из проводимости нижней ступеньки $\sigma_{d c}=5.5 \cdot 10^{-6} \Omega^{-1} \mathrm{~m}^{-1}$ в предположении о независимом движении зарядов [8]. Малое значение проводимости напрямую пересчитывается в малое значение концентрации $N_{i} \sim 10^{20} \mathrm{~m}^{-3}$.

В нашей модели частицы сильно взаимодействуют, они с пикосекундными временами сталкиваются и взаимопревращаются. Взаимодействия порождают экранировку, которая создает торможение для носителей тока и ведет к спаду проводимости $\sigma$ на низких частотах. Определение концентрации частиц в этих условиях корректно только из данных высокочастотных измерений, когда отклик проводящих частиц коррелирован. Высокая проводимость верхней полки $\sigma_{a c}=80 \Omega^{-1} \mathrm{~m}^{-1}$ [4] дает высокую эффективную концентрацию $N_{i}$ независимых носителей тока (ионов).

Отмечаем, что предлагаемый нами для жидкой воды сценарий, „the existence of many short-lived hydronium and hydroxide ions in water", допускается молекулярнодинамическими симуляциями [16].

\section{Заключение}

Рассмотрена среда, электродинамические, тепловые и транспортные свойства которой в рамках естественных предположений качественно и во многом количественно повторяют свойства жидкой воды. По охвату описываемых параметров модель превосходит другие, нам известные.

\section{Финансирование работы}

Работа выполнена при частичной финансовой поддержке гранта РФФИ-19-0200446-А.

\section{Конфликт интересов}

Авторы заявляют, что у них нет конфликта интересов.

\section{Список литературы}

[1] Pettersson L.G.M., Henchman R. H., Nilsson A. // Chem. Rev. 2016. Vol. 116. P. 7459-7462.

DOI: $10.1021 /$ acs.chemrev.6b00363

[2] Aragones J.L., MacDowell L.G., Vega C. // J. Phys. Chem. A. 2011. Vol. 115. P. 5745-5758. DOI: $10.1021 /$ jp105975c

[3] Volkov A.A., Artemov V.G., Pronin A.V. // Eur. Phys. Lett. 2014. Vol. 106. P. 46004 (1-6).

DOI: $10.1209 / 0295-5075 / 106 / 46004$ 
[4] Volkov A.A., Artemov V.G., VolkovJr A.A., Sysoev N.N. // J. Mol. Liq. 2017. Vol. 248. P. 564-568.

DOI: 10.1016/j.molliq.2017.10.071

[5] Френкель Я.И. Кинетическая теория жидкостей. Л.: Наука, 1975. 592 c.

[6] Эйзенберг Д., Кауиман В. Структура и свойства воды. Л.: Гидрометеоиздат, 1975. $280 \mathrm{c.}$

[7] Маленков Г.Г. // Журнал структурной химии. 2006. Т. 47. C. 5-35.

[8] Bockris J.O'M., Reddy A.K.N. Modern Electrochemistry. 1. Ionics. NY:: Plenum Press, 1998. 770 p.

[9] Agmon N. // J. Phys. Chem. 1996. Vol. 100. P. 1072-1080. DOI: $10.1021 / \mathrm{jp} 9516295$

[10] Ландау Л.Д., Лифиии, Е.М. Теоретическая физика. Электродинамика сплошных сред. 2-е изд. М.: Наука, 1982. $621 \mathrm{c}$.

[11] Волков А.А., Васин А.А, Волков А.А.мл. // Изв. РАН. Сер. физ. 2020. Т. 84. № 1. С. 56-60. DOI: $10.1134 / \mathrm{S} 0367676520010329$

[12] Stepisnik J., Mohoric A., Sersa I. arXiv:1010.1175v1 (2010).

[13] Volkov A.A., Vasin A.A., Volkov A.A.Jr // Ferroelectrics. 2019. Vol. 538. P. 83-88. DOI: 10.1080/00150193.2019.1569989

[14] The International Association for the Properties of Water and Steam (IAPWS). Releases. [Электронный pecypc] URL: http://www.iapws.org/release.html

[15] Okada K., Yao M., Hiejima Y., Kohno H., Kajihara Y. // J. Chem. Phys. 1999. Vol. 110. P. 3026-3036. DOI: 10.1063/1.477897

[16] Geissler Ph.L., Dellago C., Chandler D., Hutter J., Parrinello M. // Science. 2001. Vol. 291. P. 2121-2124. DOI: $10.1126 /$ science.1056991 